\title{
A LARGE-SCALE CO IMAGING OF THE GALACTIC CENTER
}

\author{
T. OKA ${ }^{1}$, T. HASEGAWA ${ }^{2}$, F. SATO $^{3}$, H. YAMASAKI ${ }^{3}$, \\ M. TSUBOI ${ }^{4}$ AND A. MIYAZAKI ${ }^{4}$ \\ ${ }^{1}$ The Institute of Physical and Chemical Research (RIKEN) \\ ${ }^{2}$ Institute of Astronomy, The University of Tokyo \\ ${ }^{3}$ Department of Astronomy and Earth Sciences, \\ Tokyo Gakugei University \\ ${ }^{4}$ Institute of Astrophysics and Planetary Science, \\ Ibaraki University
}

\section{Introduction and Observations}

Molecular gas in the Galactic center region is spatially and kinematically complex, and its physical conditions are distinctively different from those of molecular gas in the Galactic disk (e.g., Morris 1996). Relative paucity of current star formation activity, despite the abundance of dense molecular gas in this region, is one of the problem at issue.

Using the $2 \times 2$ multi-beam SIS receiver at the NRO $45 \mathrm{~m}$ telescope (beamwidth $16^{\prime \prime}$ ), we have made $\mathrm{CO}$ high resolution mapping observations of the Galactic center region. We have collected about $44,000{ }^{12} \mathrm{CO}(J=1-$ $0)$ spectra and over $13,000{ }^{13} \mathrm{CO}(J=1-0)$ spectra with $34^{\prime \prime}$ grid spacing. The ${ }^{12} \mathrm{CO}$ data cover almost the full extent of the Galactic center molecular cloud complex.

\section{Morphology and Kinematics}

Our CO images with extremely wide spatial dynamic range provide innovative view of the molecular gas in the Galactic center region (Oka et al. 1996, Hasegawa et al. 1996).

- Enormous number of molecular arcs and/or shells.

- Sharp emission edges and filamentary structures.

- An high velocity expanding molecular ring $(d \sim 50 \mathrm{pc})$ near the center.

- A molecular "smoke" originated from the central 10 parsecs.

- Large molecular flare at $l \simeq 1.3^{\circ}$ consisting of many filaments. 


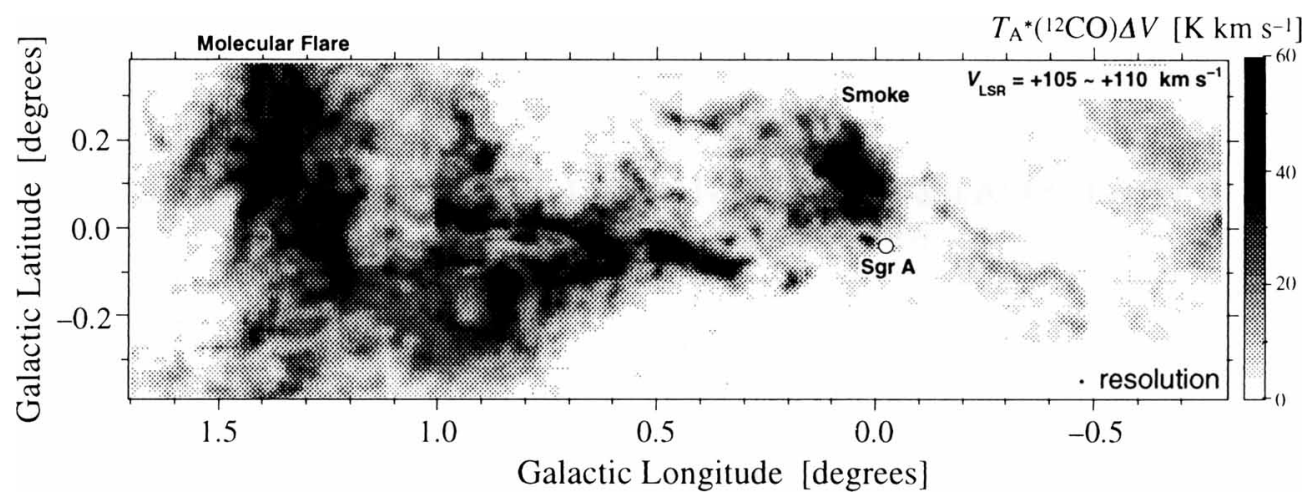

Figure 1. A gray-scale image of ${ }^{12} \mathrm{CO}(J=1-0)$ emission in the velocity range $V_{\mathrm{LSR}}=+105$ to $+110 \mathrm{~km} \mathrm{~s}^{-1}$. Molecular flare at $l \simeq 1.3^{\circ}$ is associated with a number of molecular shells/arcs.

The morphology and kinematics of molecular gas strongly suggest that there are enormous number of supernova remnants in the Galactic center region, and that the region has experienced an era of active star formation in the recent past. A burst of star formation with a short duration time will have peak in the mechanical energy released as superwind about $5 \times 10^{7}$ years after a starburst (Heckman et al. 1993). The Galactic center may be currently in a "wind dominated" phase with quiescent star formation after a starburst.

The central region of the Galaxy may have been experienced recurrent bursts of star formation. A concentration of AGB stars in the central 100 pc (Lindqvist et al. 1991) could be remnants of ancient starbursts.

\section{References}

Hasegawa, T. et al. 1996, Nature, submitted

Heckman, T. M., Lehnert, M. D., and Armus, L. 1993, in "The Environment and Evolution of Galaxies," eds. J. M. Shull and H. A. Thronson, Jr. (Dordrecht: Kluwer), 455

Lindqvist, M., Winnberg, A., Habing, H.J., and Matthews, H.E. 1991, Astron.Astrophys.Suppl., 92, 43

Morris, M. 1996, in IAU Symposium 170, "CO:Twenty-five Years of Millimeter-wave Spectroscopy," in press

Oka, T., Hasegawa, T., Sato, F., Tsuboi, M., and Handa, T. 1996, in IAU Symposium 170, "CO:Twenty-five Years of Millimeter-wave Spectroscopy," in press 